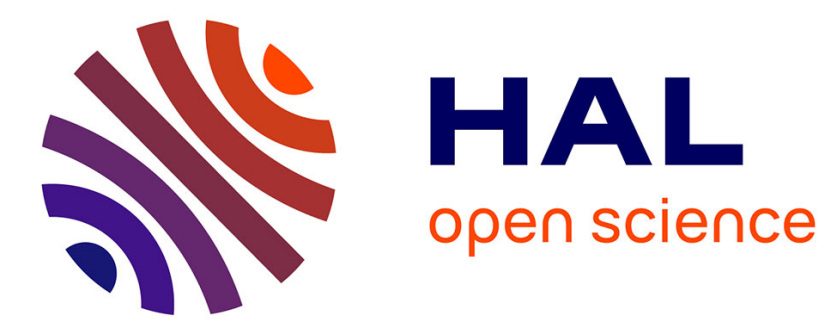

\title{
Optimal Power and Resource Allocation for Transmit Power Minimization in OFDMA-based NOMA Networks
}

Pierre Bélis, Rémi Bonnefoi, Haïfa Farès, Yves Louet

\section{To cite this version:}

Pierre Bélis, Rémi Bonnefoi, Haïfa Farès, Yves Louet. Optimal Power and Resource Allocation for Transmit Power Minimization in OFDMA-based NOMA Networks. 2019. hal-02053034

\author{
HAL Id: hal-02053034 \\ https://hal.science/hal-02053034
}

Preprint submitted on 28 Feb 2019

HAL is a multi-disciplinary open access archive for the deposit and dissemination of scientific research documents, whether they are published or not. The documents may come from teaching and research institutions in France or abroad, or from public or private research centers.
L'archive ouverte pluridisciplinaire HAL, est destinée au dépôt et à la diffusion de documents scientifiques de niveau recherche, publiés ou non, émanant des établissements d'enseignement et de recherche français ou étrangers, des laboratoires publics ou privés. 


\title{
Optimal Power and Resource Allocation for Transmit Power Minimization in OFDMA-based NOMA Networks
}

\author{
Pierre Bélis*, Rémi Bonnefoi*, Haïfa Farès* and Yves Louët* \\ ${ }^{*}$ CentraleSupélec/IETR, CentraleSupélec Campus de Rennes, 35510 Cesson-Sévigné, France \\ Email:\{pierre.belis, remi.bonnefoi, haifa.fares, yves.louet\}@centralesupelec.fr
}

\begin{abstract}
In this article, we address the problem of optimal joint power and resource allocation when Non-Orthogonal Multiple Access (NOMA) and Orthogonal Frequency Multiple Access (OFDMA) are combined for a hybrid downlink multiple access. First, an optimal solution of joint power and resource allocation minimizing the transmit power consumption, is obtained by rewriting the original optimization problem into an equivalent convex one and then by solving it by means of the well-known interior-point method. Second, we analyze the properties of the optimum, which are twofold; we show that at the optimum, the order of the users is preserved from one channel to another. Then, we derive a closed-form expression for the optimal power allocation in the particular case where all users have positive non-zero transmit powers in all channels.
\end{abstract}

\section{INTRODUCTION}

Non-Orthogonal Multiple Access (NOMA), and in particular Power-Domain NOMA (PD-NOMA) [1], has been recently proposed as a promising multiple access method for future $5 \mathrm{G}$ communication systems [2] to avert radio resource shortage due to a huge increase of the number of connected devices expected within few years. Contrary to traditional Orthogonal Multiple Access (OMA) techniques such as Orthogonal Frequency Division Multiple Access (OFDMA); several users can be served in the same channel with NOMA. By exploiting the channel gain differences, users are multiplexed into transmission power domain and then are non-orthogonally scheduled for transmission on the same spectrum resources. Successive interference cancellation (SIC) is then applied at the receivers to decode the message signals.

Recently, NOMA combined with OFDMA has been recognised to perform better than conventional OFDMA systems as well as than pure NOMA schemes. For instance, it may ensure more spectral efficiency and accommodate more users than the conventional OFDMA-based systems [3]. Moreover, joint power and resource allocation allows to OFDMA-based NOMA to outperform pure NOMA in terms of transmit power consumption. However, these benefits can be deeply affected by power and resource allocations. Therefore, the problem of joint power and resource allocation in OFDMA-based NOMA systems with the objective to minimize the transmit power consumption of a base station under a minimum capacity requirement for served users, has attracted a lot of attention in the research community.
Several solutions have been proposed in the literature in order to address this problem. In [3], the authors solve it in the case where only two users can be served in each channel. In [4], the authors present a solution in order to solve a convex approximation of the initial optimization problem. In [5], the authors propose several sub-optimal resource allocation techniques to solve this problem. In [6], it has been proven that the problem of power allocation can be rewritten in a convex form and it has been proposed a sub-optimal algorithm for resource allocation. Nevertheless, none of the above mentioned articles propose solutions in order to optimally solve the problem of power and resource allocation for minimizing the transmit power of a general model of an OFDMA-based NOMA base station.

In the present paper, the starting point is the reformulation proposed in [6] and we extend this result in order to show that the whole problem of power and resource allocation can be solved using classical convex optimization algorithms. In [6], the power and resource allocations are obtained sequentially; the authors first present an optimal solution of power consumption with fixed resource allocation and then based on this, they try to refine this solution in order to jointly optimize both power and resource allocation by proposing a near-optimal algorithm. Although we start from the same reformulation given in [6], we proceed differently by first jointly optimizing power and resource allocation assuming a unique optimization problem. Second, we carry out an analysis of the properties of the optimal power and resource allocation. The main concluding remarks that we can draw from this analysis are summarized as:

- the problem of power and resource allocation can be solved using well-known algorithms such as an interior point method [7],

- when optimal power and resource allocation is performed, the order of the users is preserved from one channel to an other; when a user order is defined for a channel, no switching is possible in another channel,

- a closed-form expression for the optimal power and resource allocation is proposed when assuming non-zero transmit power for all users in all channels.

The paper is organized as follows. The system model is 


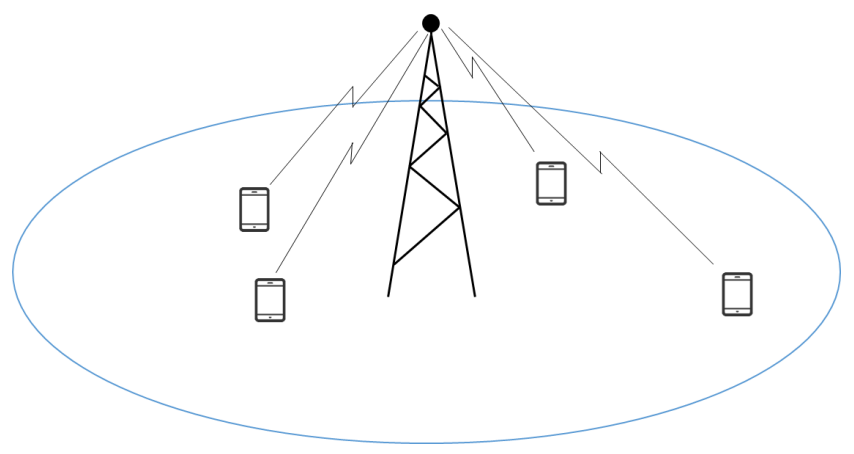

Fig. 1. The proposed system model where a base station serves $N u$ users.

briefly described in Section II. In Section III, we set the problem of joint power and resource allocation in OFDMAbased NOMA system. In Section IV, we start by proving that the problem of power and resource allocation can be solved jointly using convex optimization algorithms; then, we analyze the optimal power and resource allocation. Numerical simulations reported in Section $\mathrm{V}$ corroborate the previous analysis. Finally, conclusions are conducted in Section VI.

\section{SySTEM MOdEL}

As illustrated in Figure 1, we assume the downlink of a base station which serves $\mathrm{Nu}$ users. To do so, the base station has a bandwidth $B$ which is divided in $N_{c}$ channels of size $B_{c}=\frac{B}{N_{c}}$. We denote by $g_{k, n}=\left|h_{k, n}\right|^{2}$ the channel coefficient of the user $k$ in channel $n$. Moreover, we denote $\sigma^{2}=k_{B} T B_{c}$ the thermal noise variance, where $k_{B}$ is the Boltzmann constant and $T$ is the temperature in Kelvin. The base station aims to ensure to each user a given QoS in term of minimum capacity. We denote $C_{k}$ the capacity constraint of user $k$.

The base station combines two techniques for the multiple access. The first one is the OFDMA [8], [9], where the users are orthogonally multiplexed into the spectral domain. The second technique is NOMA [1], where several users can be multiplexed in the same channel, for example in the power domain. We give an example of the considered multiple access scheme in Figure 2.

We denote by $P_{\mathrm{Tx}}^{k, n}$ the transmit power used to serve user $k$ in channel $n$. As explained in [10], when NOMA is used in one channel, the transmit power $P_{\mathrm{Tx}}^{k, n}$ allocated to each user decreases as the channel coefficient of the user $g_{k, n}$ increases. In each channel where NOMA is used, each user employs a SIC receiver to iteratively cancel the signal which has a higher transmit power. Consequently, by exploiting this power order, each user is able to recover its own signal. Denoting $\mathscr{S}_{k}$ the set of channels allocated to user $k$, the capacity, $C_{k, n}$, of user $k$ in channel $n$ is given by

$$
C_{k, n}=B_{c} \log _{2}\left(1+\frac{P_{\mathrm{Tx}}^{k, n} g_{k, n}}{\sigma^{2}+I_{k, n}}\right),
$$

with $I_{k, n}$ is the remaining interference caused by signals transmitted to the users served in the same channel and which

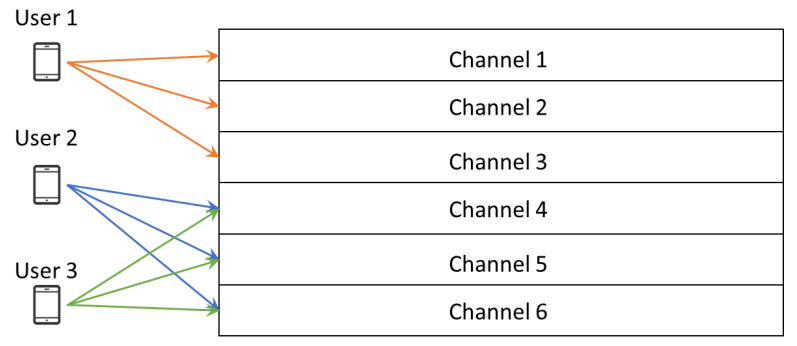

Fig. 2. An example of NOMA-OFDMA. In this example, users 1 and 2 are separated in the frequency domain (OFDMA), whereas users 2 and 3 are separated in the power domain (NOMA).

can not be removed by SIC. Assuming perfect SIC, this interference is defined as

$$
I_{k, n}=g_{k, n} \sum_{\begin{array}{c}
i \text { s.t.: } \\
\text { and, } g_{i, n}>\mathscr{S}_{i, n}
\end{array}} P_{\mathrm{Tx}}^{i, n} .
$$

\section{Problem Formulation}

The considered optimization problem aims to minimize the transmit power of the base station operating with OFDMAbased NOMA under a minimum capacity requirement for served users. Denoting $C_{k}$ the capacity constraint of user $k$, the problem to be solved in terms of both resource and power allocation, can be written as

$$
\begin{aligned}
\underset{P_{\mathrm{Tx}}^{k, n}, \mathscr{S}_{k}}{\arg \min } & \sum_{k=1}^{N u} \sum_{n \in \mathscr{S}_{k}} P_{\mathrm{Tx}}^{k, n}, \\
\text { s.t. } \quad & C_{k}-B_{c} \sum_{n \in \mathscr{S}_{k}} \log _{2}\left(1+\frac{P_{\mathrm{Tx}}^{k, n} g_{k, n}}{\sigma^{2}+I_{k, n}}\right) \leq 0, \quad \forall k, \\
& \text { where, } I_{k, n}=g_{k, n} \sum_{\begin{array}{c}
i \text { s.t.: } n \in \mathscr{S}_{i} \\
\text { and, } g_{i, n}>g_{k, n}
\end{array}} P_{\mathrm{Tx}}^{i, n}, \\
& P_{\mathrm{Tx}}^{k, n} \geq 0, \quad \forall k, n .
\end{aligned}
$$

In the problem defined by (3), (3a) is the objective function which is the sum of the transmit power of all users in their allocated channels. Besides, (3b) depicts the capacity constraints of the users and $(3 \mathrm{~d})$ is a physical constraint which ensures that all the transmit powers are positive. In the following, we propose an algorithm to optimally allocate both power and resource to the served users in order to minimize the transmit power as defined in (3a).

After assuming that the resource allocation has been done, we rewrite the problem in a more tractable form, as given in [6]. The key idea in this reformulation is to reorder the transmit powers of involved users for each channel $n$, by introducing the index $k_{n}$ which is the index of user $k$ over the channel $n$. Assuming a resource allocation hypothesis and according to the new notation, we have a set of $N u(n)$ users non-orthogonally scheduled over the channel $n$ : each of them has a channel coefficient $g_{k, n}$ and a transmit power $P_{\mathrm{Tx}}^{k, n}$ in 
channel $n$. For instance, the index $k_{n}$ is equal to 1 if user $k$ has the highest coefficient in channel $n$; it is equal to 2 if user $k$ has the second highest coefficient in channel $n$ and so on. According to this new notation, the problem of (3) becomes

$$
\begin{aligned}
\underset{P_{\mathrm{Tx}}^{k_{n}, n}}{\arg \min } & \sum_{n=1}^{N_{c}} \sum_{k_{n}=1}^{N u(n)} P_{\mathrm{Tx}}^{k_{n}, n}, \\
\text { s.t. } & C_{k}-B_{c} \sum_{n \in \mathscr{S}_{k}} \log _{2}\left(1+\frac{P_{\mathrm{Tx}}^{k_{n}, n} g_{k_{n}, n}}{\sigma^{2}+I_{k_{n}, n}}\right) \leq 0, \quad \forall k, \\
& \text { where, } I_{k_{n}, n}=g_{k_{n}, n} \sum_{i_{n}=1}^{k_{n}-1} P_{\mathrm{Tx}}^{i_{n}, n}, \\
& P_{\mathrm{Tx}}^{k_{n}, n} \geq 0, \quad \forall k, n .
\end{aligned}
$$

We now rewrite the problem of (4) as a function of $C_{k_{n}, n}$, the capacity of user $k$ in channel $n$. Using (1), the transmit power of user $k$ in channel $n$ is equal to:

$$
P_{\mathrm{Tx}}^{k_{n}, n}=\left(2^{C_{k_{n}, n} / B_{c}}-1\right)\left(\frac{\sigma^{2}}{g_{k_{n}, n}}+\sum_{i_{n}=1}^{k_{n}-1} P_{\mathrm{Tx}}^{i_{n}, n}\right) .
$$

Let's introduce $\Gamma_{k_{n}, n}=\sum_{i_{n}=1}^{k_{n}} P_{\mathrm{Tx}}^{i_{n}, n}$ as the sum of the transmit powers lower or equal to $P_{\mathrm{Tx}}^{k_{n}, n}$. With this new notation, (5) becomes:

$$
\Gamma_{k_{n}, n}=\left(2^{C_{k_{n}, n} / B_{c}}-1\right)\left(\frac{\sigma^{2}}{g_{k_{n}, n}}+\Gamma_{k_{n}-1, n}\right)+\Gamma_{k_{n}-1, n},
$$

where $\Gamma_{0, n}=0$. Subsequently, we can express $\Gamma_{k_{n}, n}$ as a function of the capacity of the users in channel $n$ :

$$
\begin{aligned}
\Gamma_{k_{n}, n} & =\sum_{j_{n}=1}^{k_{n}-1}\left(\sigma^{2} \frac{2^{\frac{C_{j_{n}, n}}{B_{c}}}-1}{g_{j_{n}, n}} 2^{\frac{\sum_{l_{n}=j_{n}+1}^{k_{n}} C_{l_{n}, n}}{B_{c}}}\right) \\
& +\frac{\sigma^{2}}{g_{k_{n}, n}}\left(2^{\frac{C_{k_{n}, n}}{B_{c}}}-1\right) .
\end{aligned}
$$

We can now express the minimization problem of (4) as a function of the variables $C_{k_{n}, n}$

$$
\begin{aligned}
\underset{C_{k_{n}, n}}{\arg \min } & \sum_{n=1}^{N_{c}} \Gamma_{N u(n), n}, \\
\text { s.t. } & C_{k}-\sum_{n \in \mathscr{S}_{k}} C_{k_{n}, n} \leq 0, \quad \forall k, \\
& C_{k_{n}, n} \geq 0, \quad \forall k, n .
\end{aligned}
$$

It has been proved in [6] that the problem of power allocation defined by (8) is convex. So it can be solved using an algorithm such as an interior point method [7].

\section{OPTIMAL POWER AND RESOURCE ALLOCATION}

In this section, we first show that the problem of resource allocation can be solved using a convex optimization algorithm. Then, we emphasize some properties of the optimal power and resource allocation when all the users have a non-zero transmit power in all the channels.

\section{A. Optimal resource allocation}

The problem defined by (8) is convex for any resource allocation [6]. And so it is, when all users are allocated to all channels. In that case, as the problem of power allocation is convex, we know that the use of a convex optimization algorithm provides the optimal result. This algorithm starts by allocating to all users all channels and then it may set some transmit powers of some users over some subchannels to zero, when providing the optimal power allocation; this consists in an implicit optimal resource allocation result. Thus, this algorithm provide the optimal joint power and resource allocation result.

In other words, we can perform the optimal power and resource allocation by starting to allocate to all users all channels and to perform an optimal power allocation employing any convex optimization algorithm [11], while zero transmit powers are possible solutions (in this case, the channel is not assigned to this particular user).

\section{B. Order of users at the optimum}

The following property characterizes the optimal power allocation:

Proposition 1. Assuming $k$ and $k^{\prime}$ two users; and $n_{1}$ and $n_{2}$ two channels such that $g_{k, n_{1}}>g_{k^{\prime}, n_{1}}$ and $g_{k, n_{2}}<g_{k^{\prime}, n_{2}}$. If $P_{T x}^{k, n_{1}}>0$ and $P_{T x}^{k^{\prime}, n_{1}}>0$, then either $P_{T x}^{k, n_{2}}=0$ or $P_{T x}^{k^{\prime}, n_{2}}=0$.

The proof is given in appendix A.

In other words, in the case where two users are served in a given order in one channel in terms of transmit power (one with a higher transmit power than the other one), they cannot be served in another channel in the reverse order.

Thanks to this proposition, we can anticipate some of the transmit powers that are going to be set to zero. To do so, we have to sort the users in a given order, e.g., by descending order of their average channel gain (or pathloss). Once the users are sorted, user 1 is the one with the highest pathloss, and user $N_{u}$ is the one with the lowest pathloss.

\section{A particular case: when all users have a non-zero transmit power in all channels}

In order to better understand the optimal power allocation, we derive, in this section, its expression in the case where all users are served with a non-zero transmit power in all channels. It is worth noting that, according to Proposition 1, this case occurs only when the order of users is preserved between channels. Thus, we have, for all $k \in \llbracket 1 ; N_{u} \rrbracket$, $k=k_{1}=k_{2}=\cdots=k_{N_{c}}$ and $N_{u}(n)=N_{u}, \forall n \in \llbracket 1 ; N_{c} \rrbracket$.

Proposition 2. In the case where all users have a non-zero transmit power in all channels, the optimal capacity of user $k$ in channel $n$ is given by: 


$$
\begin{gathered}
C_{N u, n}=\frac{C_{N u}}{N_{c}}-\frac{B_{c}}{N_{c}} \sum_{m=1}^{N_{c}} \log _{2}\left(\frac{g_{N u-1, m} g_{N u, m}}{\left(g_{N u-1, m}-g_{N u, m}\right) \frac{\sigma^{2} \ln (2)}{B_{c}}}\right) \\
+B_{c} \log _{2}\left(\frac{g_{N u-1, n} g_{N u, n}}{\left(g_{N u-1, n}-g_{N u, n}\right) \frac{\sigma^{2} \ln (2)}{B_{c}}}\right), \\
C_{k, n}=\frac{C_{k}}{N_{c}}-\frac{B_{c}}{N_{c}} \sum_{m=1}^{N_{c}} \log _{2}\left(\frac{g_{k-1, m}\left(g_{k, m}-g_{k+1, m}\right)}{g_{k+1, m}\left(g_{k-1, m}-g_{k, m}\right)}\right) \\
+B_{c} \log _{2}\left(\frac{g_{k-1, n}\left(g_{k, n}-g_{k+1, n}\right)}{g_{k+1, n}\left(g_{k-1, n}-g_{k, n}\right)}\right),
\end{gathered}
$$

$C_{1, n}=\frac{C_{1}}{N_{c}}-\frac{B_{c}}{N_{c}} \sum_{m=1}^{N_{c}} \log _{2}\left(\frac{g_{1, m}-g_{2, m}}{g_{2, m}}\right)+B_{c} \log _{2}\left(\frac{g_{1, n}-g_{2, n}}{g_{2, n}}\right)$.

The formulas proposed here are not valid in the general case. Indeed, when the index of users varies from one channel to another (i.e. $k_{n} \neq k_{n^{\prime}}$ ), it is impossible to obtain as clear closed-form expressions as the ones presented in Proposition 2. As these formulas are only valid in a particular case, they cannot be used for power allocation in any case. However, the result given by these formulas can be used as a starting point for the interior point method. To the best of our knowledge, unlike the OFDMA case where the water-filling formula is always valid, we did not find a general formula for the NOMA case. Besides, the transmit power of user 1 is:

$$
P_{\mathrm{Tx}}^{1, n}=\frac{\sigma^{2}\left(g_{1, n}-g_{2, n}\right)}{g_{2, n} g_{1, n}}\left(\frac{2^{\frac{C_{1}}{N_{c} B_{c}}}}{\left(\prod_{m=1}^{N_{c}} \frac{g_{1, m}-g_{2, m}}{g_{2, m}}\right)^{\frac{1}{N_{c}}}}-\frac{g_{2, n}}{g_{1, n}-g_{2, n}}\right)
$$

By studying this particular case, we observe that, in the case where all users have a non-zero transmit power in all channels, the expression of the transmit powers of user 1 is similar to the well-known water-filling algorithm [9]. Moreover, we can see that compared to the OFDMA case where the power allocation depends on the channel coefficients, in the NOMA case, the transmit power is dependent on the difference between the channel coefficients.

\section{Simulation Results}

In this section, numerical results obtained from simulations are given in order to assess the performance of the proposed optimal joint power and resource allocation when OFDMAbased NOMA is employed. We consider a micro-cell that serves several users. As in the LTE standards we suppose that the band is divided in $N_{c} 180 \mathrm{kHz}$ bandwidth subchannels. The thermal noise in each channel is equal to $\sigma^{2}=-121.44 \mathrm{~dB}$ and a $2 \mathrm{~dB}$ noise figure is considered. Moreover, we consider a $10 \mathrm{dBi}$ antenna gain and the pathlosses are computed with the Winner II 'B1 - Urban Micro-cell' pathloss model with a $2 \mathrm{GHz}$ central frequency. We employ the Extended Typical

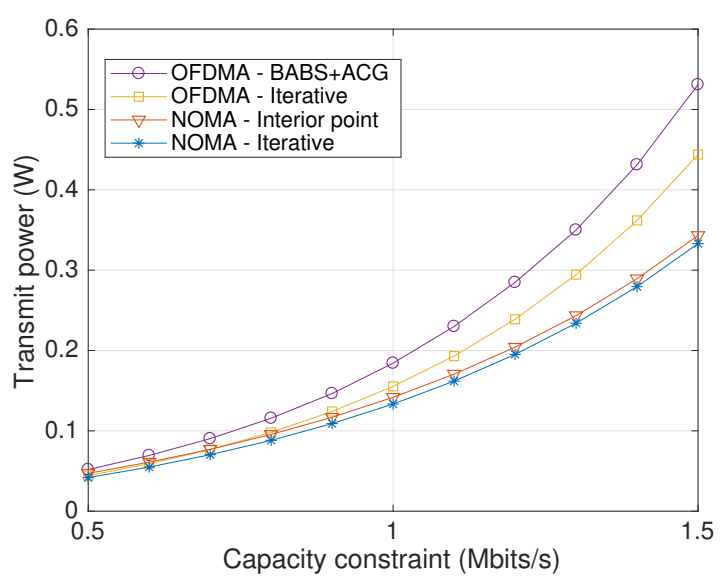

Fig. 3. Total transmit power of the base station when both users are at the same distance from the base station(equal to $400 \mathrm{~m}$ ).

Urban (ETU) channel model to generate fading. We suppose that all users have the same capacity constraint.

We first consider $N_{c}=4$ channels and $N_{u}=2$ users. In such a configuration, with few channels and users, we can compare the proposed solution with iterative approaches. Therefore, for sake of fairness, we compare:

1) The proposed solution where the optimal joint power and resource allocation is computed using an interior point method. This scheme is denoted in figures by NOMA Interior point.

2) The optimal OFDMA-based NOMA where the resource allocation is performed through an exhaustive search and the optimal power allocation is conducted using an interior point method. Correspondingly, this scheme is denoted by NOMA - iterative.

3) The optimal OFDMA power and resource allocation where the resource allocation is performed through an exhaustive search and the power allocation by means of water-filling algorithm. Correspondingly, this scheme is denoted by OFDMA - iterative.

4) A sub-optimal power and resource allocation from [8] where the Bandwidth Assignment Based on SNR (BABS) and Amplitude Craving Greedy (ACG) are respectively used for computing the number of channels per user and for channels assignment, and, where the power allocation is done with the water-filling algorithm.

We first consider that the two users are at the same distance from the base station (equal to $400 \mathrm{~m}$ ), and, we display in Figure 3 the transmit power of the base station versus the capacity constraint of the users.

In the case where the users are at the same distance from the base station, OFDMA-based NOMA schemes offer sensible gains but not sizable ones compared to OFDMA schemes. Indeed, when the users have a similar pathloss, some inversions between the channel coefficients can occur, and consequently, according to Proposition 1, only one user is served 


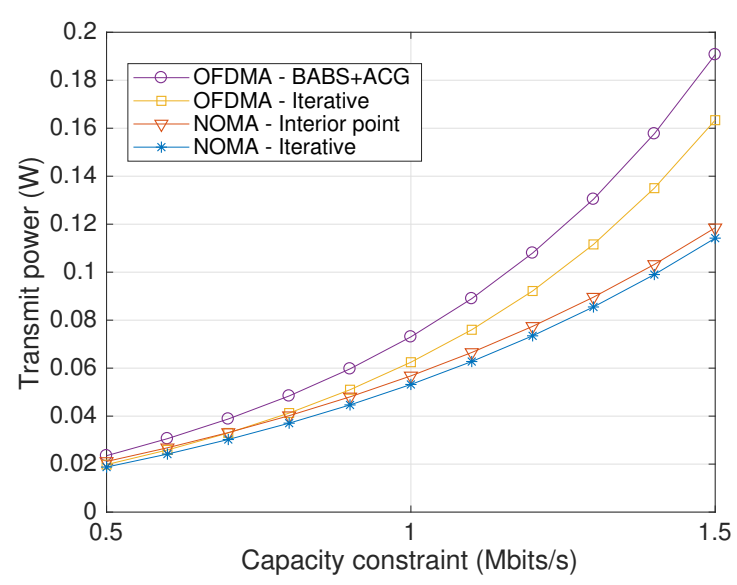

Fig. 4. Total transmit power of the base station when one user is near the base station and the other far.

in some channels. In that case, the iterative NOMA strategy provides between 5 and $25 \%$ transmit power gain. Besides, the proposed strategy provides up to $35 \%$ transmit power gain compared to the BABS+ACG strategy. Moreover, we can see in Figure 3 that there is a small gap between NOMA with an exhaustive resource allocation and the proposed strategy. This difference is to be attributed to numerical approximations.

We now consider the case where the first user is near the base station $(200 \mathrm{~m})$ and the second is far from it $(400 \mathrm{~m})$. The results are displayed in Figure 4. In this scenario where the difference between the channel coefficients is important, OFDMA-based NOMA scheme have larger gains compared to OFDMA. In that case, the transmit power consumption with the proposed strategy is up to $40 \%$ lower than that required with the BABS+ACG policy.

We now consider a more realistic scenario where the base station has a $500 \mathrm{~m}$ cell radius. Moreover, 50 channels are used to serve users. We first consider that $N_{u}=10$ uniformly distributed users within the Cell coverage and served by the base station. The results are reported in Figure 5. We observe that the benefit of OFDMA-based NOMA over pure OFMDA increases with the capacity constraint of the users. In the simulated scenario, the gain provided by OFDMA-based NOMA varies from 6 and $50 \%$.

We now analyse the evolution of the total transmit power as a function of the number of users in the cell coverage. For that purpose, we consider that each user as a capacity constraint of $1 \mathrm{Mbits} / \mathrm{s}$ and we display in Figure 6 the total transmit power of the base station as a function of $N_{u}$. We observe in this figure that the benefit of OFDMA-based NOMA compared to pure OFDMA increases with the number of users. This is an expected and non-surprizing result since pure OFDMA can only have one user per channel, which limits its potential, mainly when the number of users is close to the number of channels. In the case where $N_{u}=15$ users are served by the base station, OFDMA-based NOMA has a $20 \%$ transmit power gain compared to pure OFDMA. To have the same

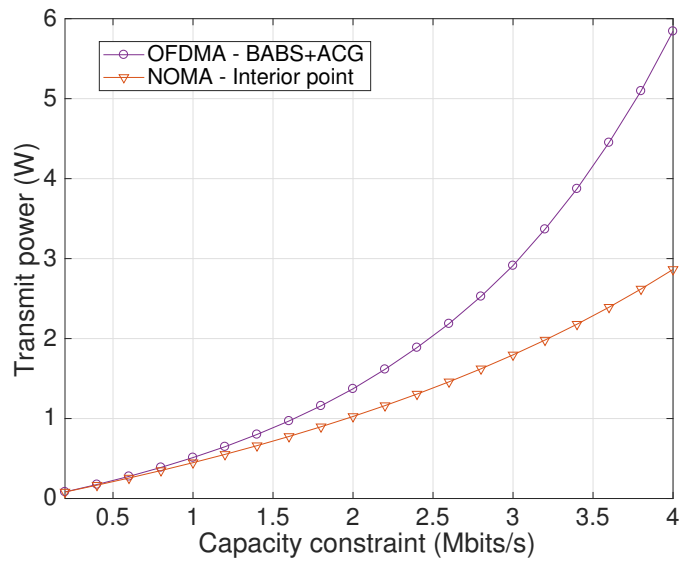

Fig. 5. Comparison of the proposed NOMA strategy with an efficient OFDMA strategy [8] versus the capacity constraint of the users.

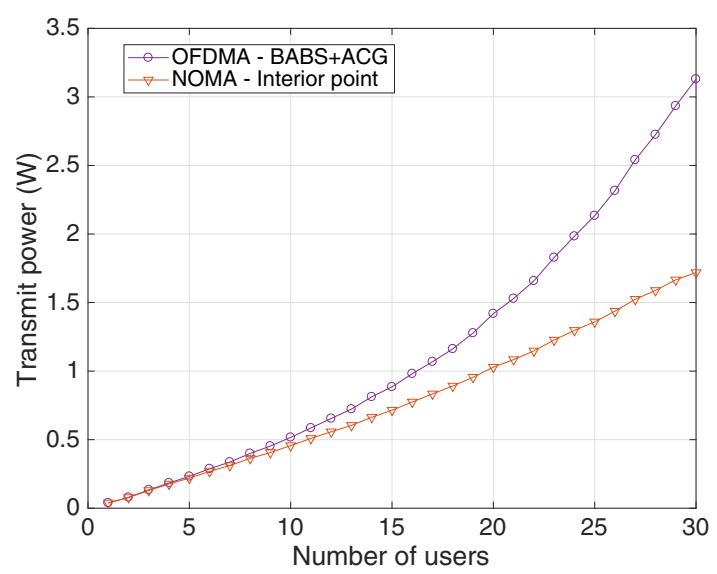

Fig. 6. Comparison of the proposed OFDMA-based NOMA strategy with an efficient OFDMA strategy [8] versus the number of users in the cell coverage.

performance in OFDMA, we have to use twice as much channels.

\section{CONCLUSIONS}

In this paper, we have shown that the problem of power and resource allocation in a OFDMA-based NOMA systems can be solved using an interior point method. Then, we have analyzed the optimum. We have shown that at the optimum, the users are served in a given order in terms of transmit powers which does not change from one channel to another. Our numerical results show that with the proposed power and resource allocation, OFDMA-based NOMA outperforms pure OFDMA.

The interior point method can be long to converge and can lead to some numerical approximation errors, mainly when several constraints are binding. That is why, in our future work, we plan to propose sub-optimal solutions for the purpose of power and resource allocation in OFDMA-based NOMA systems. 


\section{ACKNOWLEDGEMENT}

This work has been performed in the framework of the WONG5 project, receiving funds from the French National Research Agency (ANR) under the contract number ANR-15CE25-0005-02.

\section{APPENDIX A}

PROOF OF PROPOSITION 1

The Lagrangian of the problem defined by (8) is equal to

$$
\mathscr{L}=\sum_{n=1}^{N_{c}} \Gamma_{N u(n), n}-\sum_{k=1}^{N u} \lambda_{k}\left(\sum_{n \in \mathscr{S}_{k}} C_{k_{n}, n}-C_{k}\right),
$$

where $\lambda_{k}$ is the Lagrange multiplier relative to the capacity constraint of user $k$. It is worthnoting that, the constraints of positivity are not considered in the Lagrangian and will be considered later. Since the problem defined by (8) is convex, the gradient of this Lagrangian is zero at the optimum, i.e.,

$$
\frac{\partial \mathscr{L}}{\partial C_{k_{n}, n}}=0 \Leftrightarrow \frac{\partial \Gamma_{N u(n), n}}{\partial C_{k_{n}, n}}=\lambda_{k} .
$$

Then, in a channel which is used to serve at least two users, the partial derivative of $\Gamma_{N u(n), n}$ with respect to $C_{k_{n}, n}, k_{n}>1$, is

$$
\begin{aligned}
& \frac{\partial \Gamma_{N u(n), n}}{\partial C_{k_{n}, n}}=\frac{\sigma^{2} \ln (2) 2^{\sum_{l_{n}=1}^{N u(n)} \frac{C_{l_{n}, n}}{B_{c}}}}{B_{c} g_{1, n}}+ \\
& \sum_{j_{n}=1}^{k_{n}-1}\left(\frac{\sigma^{2} \ln (2)}{B_{c} g_{j_{n}, n} g_{j_{n}+1, n}}\left(g_{j_{n}, n}-g_{j_{n}+1, n}\right) 2^{\sum_{l_{n}=j_{n}+1}^{N u(n)} \frac{C_{l_{n}, n}}{B_{c}}}\right) .
\end{aligned}
$$

Expression in (15) can be rewritten as

$$
\begin{aligned}
& \frac{\partial \Gamma_{N u(n), n}}{\partial C_{k_{n}, n}}=\frac{\partial \Gamma_{N u(n), n}}{\partial C_{k_{n}-1, n}}+ \\
& \frac{\sigma^{2} \ln (2)}{B_{c} g_{k_{n}, n} g_{k_{n}-1, n}}\left(g_{k_{n}-1, n}-g_{k_{n}, n}\right) 2^{\sum_{l_{n}=k_{n}}^{N u(n)} \frac{C_{l_{n, n}, n}}{B_{c}}} .
\end{aligned}
$$

Putting (14) in (16), we derive

$$
\begin{aligned}
\lambda_{k_{n}, n}- & \lambda_{k_{n}-1, n}= \\
& \frac{\sigma^{2} \ln (2)}{B_{c} g_{k_{n}, n} g_{k_{n}-1, n}}\left(g_{k_{n}-1, n}-g_{k_{n}, n}\right) 2^{\sum_{l_{n}=k_{n}}^{N u(n)} \frac{C_{l_{n, n}}}{B_{c}}} .
\end{aligned}
$$

In (17), $\lambda_{k_{n}, n}=\lambda_{k}$ is the Lagrange multiplier of user $k$ which has index $k_{n}$ in channel $n$. Moreover, $\lambda_{k_{n}-1, n}$ is the Lagrange multiplier of the user $\left(k^{\prime}\right)$ with a directly lower transmit power in this channel. The right side of equality (17) is strictly positive, consequently, $\lambda_{k}=\lambda_{k_{n}, n}>\lambda_{k^{\prime}}=\lambda_{k_{n}-1, n}$. Subsequently, at the optimum, the Lagrange multiplier of a user is higher than the ones of the users with a lowest, but non-zero, transmit power in this channel. In other words, if the users $k$ and $k^{\prime}$ are optimally served in one channel $\left(n_{1}\right)$ with a non-zero transmit power, and where, $0<P_{\mathrm{Tx}}^{k, n_{1}}<P_{\mathrm{Tx}}^{k^{\prime}, n_{1}}$ $\left(g_{k, n_{1}}>g_{k^{\prime}, n_{1}}\right)$. Their Lagrange multipliers are going to satisfy the condition $\lambda_{k}>\lambda_{k^{\prime}}$
Consequently, if there is another channel $\left(n_{2}\right)$ where $g_{k, n_{2}}<$ $g_{k^{\prime}, n_{2}}$ the transmit power of at least one of these two users is equal to zero in channel $n_{2}$. This proves Proposition 1 .

\section{APPENDIX B}

\section{PROOF OF PROPOSITION 2}

We recall here that, all users have non-zero transmit powers. In such case, $\forall k \in \llbracket 1 ; N_{u} \rrbracket, k=k_{1}=\cdots=k_{N_{c}}$ and $N_{u}(n)=$ $N_{u}, \forall n \in \llbracket 1 ; N_{c} \rrbracket$. Thus, we can derive from equation (16)

$$
C_{N u, n}=B_{c} \log _{2}\left(\frac{\left(\lambda_{N u, n}-\lambda_{N u-1, n}\right) g_{N u-1, n} g_{N u, n}}{\left(g_{N u-1, n}-g_{N u, n}\right) \frac{\sigma^{2} \ln (2)}{B_{c}}}\right) .
$$

Then, we can deduce, $\forall k \in \llbracket 2 ; N u-1 \rrbracket$, that

$$
C_{k, n}=B_{c} \log _{2}\left(\frac{\left(\lambda_{k, n}-\lambda_{k-1, n}\right) g_{k-1, n}\left(g_{k, n}-g_{k+1, n}\right)}{g_{k+1, n}\left(g_{k-1, n}-g_{k, n}\right)}\right) .
$$

Using (15) in the case where $k=2$ and the partial derivative of the Lagrangian with respect to $C_{1, n}$, we can deduce

$$
C_{1, n}=B_{c} \log _{2}\left(\frac{\lambda_{1, n}\left(g_{1, n}-g_{2, n}\right)}{\left(\lambda_{2, n}-\lambda_{1, n}\right) g_{2, n}}\right) .
$$

For this particular case, the channel coefficients have to respect the following conditions. Every user is allocated to every channel and the Proposition 1 is respected. As every user is allocated to each channel, we have

$$
C_{k}=\sum_{m=1}^{N_{c}} C_{k, m}, \quad \forall k \in \llbracket 1 ; N u \rrbracket .
$$

Using all (18), (19), (20), and (21), we derive the three entities

$$
\begin{aligned}
\log _{2}\left(\lambda_{N u, n}-\lambda_{N u-1, n}\right) & =\frac{C_{N u}}{N_{c} B_{c}} \\
- & \frac{1}{N_{c}} \sum_{m=1}^{N_{c}} \log _{2}\left(\frac{g_{N u-1, m} g_{N u, m}}{\left(g_{N u-1, m}-g_{N u, m}\right) \frac{\sigma^{2} \ln (2)}{B_{c}}}\right),
\end{aligned}
$$

$$
\begin{aligned}
\log _{2} \frac{\left(\lambda_{k, n}-\lambda_{k-1, n}\right)}{\left(\lambda_{k+1, n}-\lambda_{k, n}\right)}=\frac{C_{k}}{N_{c} B_{c}} \\
-\frac{1}{N_{c}} \sum_{m=1}^{N_{c}} \log _{2}\left(\frac{g_{k-1, m}\left(g_{k, m}-g_{k+1, m}\right)}{g_{k+1, m}\left(g_{k-1, m}-g_{k, m}\right)}\right),
\end{aligned}
$$

$$
\log _{2} \frac{\lambda_{1, n}}{\left(\lambda_{2, n}-\lambda_{1, n}\right)}=\frac{C_{1}}{N_{c} B_{c}}-\frac{1}{N_{c}} \sum_{m=1}^{N_{c}} \log _{2}\left(\frac{g_{1, m}-g_{2, m}}{g_{2, m}}\right) .
$$

Finally, using the later results, we derive the expression of the capacity of each user in each channel given in Proposition 2 . 


\section{REFERENCES}

[1] Y. Saito, Y. Kishiyama, A. Benjebbour, T. Nakamura, A. Li, and K. Higuchi, "Non-Orthogonal Multiple Access (NOMA) for Cellular Future Radio Access," in 2013 IEEE 77th Vehicular Technology Conference (VTC Spring), pp. 1-5, June 2013.

[2] L. Dai, B. Wang, Y. Yuan, S. Han, C. I, and Z. Wang, "Non-orthogonal multiple access for $5 \mathrm{~g}$ : solutions, challenges, opportunities, and future research trends," IEEE Communications Magazine, vol. 53, pp. 74-81, September 2015.

[3] P. Parida and S. S. Das, "Power allocation in ofdm based noma systems: A dc programming approach," in 2014 IEEE Globecom Workshops (GC Wkshps), pp. 1026-1031, Dec 2014.

[4] R. Dawadi, S. Parsaeefard, M. Derakhshani, and T. Le-Ngoc, "Powerefficient resource allocation in noma virtualized wireless networks," in 2016 IEEE Global Communications Conference (GLOBECOM), pp. 16, Dec 2016

[5] J. Farah, E. Sfeir, C. A. Nour, and C. Douillard, "New resource allocation techniques for base station power reduction in orthogonal and non-orthogonal multiplexing systems," in 2017 IEEE International
Conference on Communications Workshops (ICC Workshops), pp. 618624, May 2017.

[6] X. Li, C. Li, and Y. Jin, "Dynamic resource allocation for transmit power minimization in ofdm-based noma systems," IEEE Communications Letters, vol. 20, pp. 2558-2561, Dec 2016

[7] F. A. Potra and S. J. Wright, "Interior-point methods," Journal of Computational and Applied Mathematics, vol. 124, no. 1, pp. 281 - 302, 2000. Numerical Analysis 2000. Vol. IV: Optimization and Nonlinear Equations.

[8] D. Kivanc, G. Li, and H. Liu, "Computationally efficient bandwidth allocation and power control for ofdma," IEEE Transactions on Wireless Communications, vol. 2, pp. 1150-1158, Nov 2003.

[9] K. Seong, M. Mohseni, and J. M. Cioffi, "Optimal resource allocation for ofdma downlink systems," in 2006 IEEE International Symposium on Information Theory, pp. 1394-1398, July 2006.

[10] Z. Ding, Y. Liu, J. Choi, Q. Sun, M. Elkashlan, C. L. I, and H. V. Poor, "Application of Non-Orthogonal Multiple Access in LTE and 5G Networks," IEEE Communications Magazine, vol. 55, pp. 185-191, February 2017.

[11] S. Boyd and L. Vandenberghe, Convex Optimization. New York, NY, USA: Cambridge University Press, 2004. 